\title{
Spectral linewidths of Josephson oscillators
}

\section{Salerno, M; Samuelsen, Mogens Rugholm; Yulin, AV}

\section{Published in:}

Physical Review Letters

Link to article, DOI:

10.1103/PhysRevLett.86.5397

Publication date:

2001

\section{Document Version}

Publisher's PDF, also known as Version of record

Link back to DTU Orbit

Citation (APA):

Salerno, M., Samuelsen, M. R., \& Yulin, AV. (2001). Spectral linewidths of Josephson oscillators. Physical Review Letters, 86(23), 5397-5400. https://doi.org/10.1103/PhysRevLett.86.5397

\section{General rights}

Copyright and moral rights for the publications made accessible in the public portal are retained by the authors and/or other copyright owners and it is a condition of accessing publications that users recognise and abide by the legal requirements associated with these rights.

- Users may download and print one copy of any publication from the public portal for the purpose of private study or research.

- You may not further distribute the material or use it for any profit-making activity or commercial gain

- You may freely distribute the URL identifying the publication in the public portal

If you believe that this document breaches copyright please contact us providing details, and we will remove access to the work immediately and investigate your claim 


\title{
Spectral Linewidths of Josephson Oscillators
}

\author{
M. Salerno* and M. R. Samuelsen \\ Department of Physics, The Technical University of Denmark, DK-2800 Kogens Lyngby, Denmark
}

A. V. Yulin

Institute for Physics of Microstructures of RAS, Nizhny Novgorod, 603600, Russia

(Received 30 January 2001)

\begin{abstract}
We show that the linewidth of a Josephson flux-flow oscillator has the same functional dependence on temperature, static, and dynamic resistances as the ones of Josephson single-fluxon oscillators and small Josephson junctions. This suggests a universal formula for the linewidth of Josephson oscillators.
\end{abstract}

DOI: 10.1103/PhysRevLett.86.5397

Josephson flux-flow oscillators (FFO), e.g., long onedimensional Josephson junctions operating in the flux-flow regime, represent ideal devices for practical applications in superconducting $\mathrm{mm}$ and sub-mm-wave electronics [1-5]. This is mainly due to their properties of easy and wideband tunability, high output power, and relatively narrow spectral linewidth. In contrast to Josephson single-fluxon oscillators [6], flux-flow devices operate in the presence of a magnetic field sufficiently high to induce a uniform increase of the phase along the junction (background rotation) with small amplitude excitations traveling on top of it (flux-flow dynamics). In a particlelike picture, this can be seen as a unidirectional motion of Josephson vortices (fluxons), each carrying one quantum of magnetic flux $\phi_{0}=h / 2 e$, continuously generated at one end of the junction and destroyed at the other. When the excitations collide with the junction edges, electromagnetic radiation is emitted and a time dependent signal, reflecting the spatial flux-flow dynamics inside the junction, is generated. The presence of thermal fluctuations in the current which flows through the junction introduces noise in this dynamics which then results in a linewidth in the spectrum of the emitted radiation. In recent papers by Koshelets et al. [5], it was shown that the experimentally measured linewidth of a Josephson FFO could be well fitted by the analytical expression

$$
\Delta \nu=\rho \frac{4 \pi k_{B} T}{\phi_{0}^{2}} \frac{R_{D}^{2}}{R_{S}}
$$

with the prefactor $\rho \approx 8$. In this formula $R_{S}, R_{D}, k_{B}$, and $T$ are, respectively, the static resistance (e.g., $V_{d c} / I_{d c}$ ), the dynamic resistance (e.g., $d V_{d c} / d I_{d c}$ in the bias point), the Boltzmann constant, and the temperature. On the other hand, it is known that Eq. (1) with $\rho=1$ coincides with the linewidth of a small Josephson junction [6-8], and with $\rho=1 / 4$ coincides with the expression of the linewidth of a Josephson single-fluxon oscillator [9] (in this case the extra $1 / 4$ factor is due to a modified Josephson frequencyvoltage relation). This suggests Eq. (1) to be a universal formula for the linewidth of a Josephson oscillator.

The aim of this Letter is to provide a theoretical evidence for the validity of this statement. Since the linewidths of
PACS numbers: $85.25 . \mathrm{Cp}, 05.45 . \mathrm{Yv}, 74.50 .+\mathrm{r}$

small junctions and single-fluxon oscillators are known to reduce to the above formula, we shall concentrate only on the linewidth of a Josephson flux-flow oscillator, which apparently seems to deviate from it. In some recent papers $[10,11]$, indeed, different expressions of the FFO's linewidth were obtained by using the particlelike description of the fluxon dynamics inside the junction. In these approaches, however, the linewidth was given in terms of quantities which apparently are not related to the dynamic and static resistances, so that Eq. (1) was missed. On the other hand, by using a quasilinear mode analysis for the flux-flow dynamics, we show that the linewidth expression in Eq. (1) naturally follows with the same $\rho$ as for the small junction case (i.e., $\rho=1$ ) . This result deviates from the experimental measurements of Ref. [5] by a factor $\approx 8$. Possible explanations for this discrepancy are given at the end.

We remark that a quasilinear analysis can be justified by the fact that for the flux-flow regime the nonlinearity of the system is effectively reduced by the background rotation induced by the magnetic field. In the absence of noise, this approach was used to describe the current-voltage (IV) characteristics of a FFO, both in the presence and in the absence of microwave fields [12-14].

The most remarkable property of Eq. (1) is that it contains only physical quantities which are directly measurable from the IV curve, thus making it very useful from an experimental point of view. Moreover, some of the terms entering this formula can easily be justified. Thus, the presence of $R_{D}^{2}$ can be understood as a noise conversion factor from the spectrum of the current $S_{I}(\omega)$ to the spectrum of the voltage, according to $S_{V}(\omega) \sim R_{D}^{2} S_{I}(\omega)$. Similarly, the factor $1 / \phi_{0}^{2}$ arises from the conversion of the voltage spectrum to the frequency one. On the other hand, the appearance of $R_{S}$ in Eq. (1) is not trivial and requires, as we show in the following, a careful analysis of the junction dynamics both in its high and low frequency components.

From a physical point of view one can say that the thermal fluctuations in the current induce noise amplitude and phase modulations of the voltage at the end of the junction which, in normalized units, can be represented in the form 


$$
\Phi_{t}(t)=\omega+\tilde{\chi}_{t}+\tilde{A}(t) F[\omega t+\tilde{\chi}(t)],
$$

where $F(\cdots)$ is a $2 \pi$ periodic function of unitary amplitude and $\tilde{\chi}$ is a time dependent phase (here and in the following tildes denote stochastic signals). From frequency modulation theory [15] it is known that the full half-power linewidth of a voltage signal like (2) can be expressed in terms of the power spectrum $S_{\tilde{\chi}_{t}}$ of the instantaneous frequency as

$$
2 \pi \Delta \nu=S_{\tilde{\chi}_{t}}(0)
$$

This relation is valid in the Lorentzian limit, i.e., if $S_{\tilde{\chi}_{t}}(0) \ll 2 \beta$, where $\beta$ is the characteristic frequency of the spectrum [roughly given by the damping constant $(\alpha)$ in the system], and in the following it is assumed to be satisfied. The derivation of the linewidth is then reduced to the problem of computing the low frequency part of the spectrum of $\tilde{\chi}_{t}$. To this end we need to know the phase difference $\Phi(x, t)$ across a junction in the presence of thermal noise and with a magnetic field $\kappa$ applied at the edges. As is well known [6], this is described by the perturbed sine-Gordon equation

$$
\Phi_{x x}-\Phi_{t t}=\sin (\Phi)+\alpha \Phi_{t}-\eta+\tilde{n}(x, t),
$$

with boundary conditions $\Phi_{x}(0, t)=\Phi_{x}(l, t)=\kappa$. In this equation space and time are normalized to the Josephson penetration depth $\lambda_{J}=\left(\hbar / 2 e J \mu_{0} d\right)^{1 / 2}$ and to the inverse plasma frequency $\omega_{J}^{-1}=(2 e J / \hbar C)^{-1 / 2}$, respectively, $\eta$ is the bias current normalized to $A J, \kappa$ is the magnetic field normalized to $J \lambda_{J}, \alpha$ is the shunt damping normalized to $\hbar \omega_{J} R^{-1} / 2 e A J$, and $\tilde{n}(x, t)$ is Johnson-Nyquist white Gaussian noise

$$
\left\langle\left\langle\tilde{n}(x, t) \tilde{n}\left(x^{\prime}, t^{\prime}\right)\right\rangle\right\rangle=16 \alpha \frac{k_{B} T}{E_{0}} \delta\left(x-x^{\prime}\right) \delta\left(t-t^{\prime}\right) .
$$

Here we denoted $J$ as the maximum Josephson current density, $A$ as the area of the junction, $d$ as the magnetic thickness of the oxide layer, and $C$ and $R^{-1}$ as the capacitance and the normal-state conductance per unit area, respectively. Moreover, we used $\langle\langle\rangle\rangle$ to denote the ensemble average, and $E_{0}$, the fluxon rest energy, to fix the scale of energy in the system. In the absence of noise, a solution of Eq. (4) can be obtained in the form

$$
\Phi(x, t)=\Psi_{0}+\Psi(x, t),
$$

with $\Psi(x, t)$ a small modulation $(\Psi \ll 1)$ around the rotating background $\Psi_{0}=\omega t+\kappa x+\Phi_{0}\left(\Phi_{0}\right.$ denoting an arbitrary phase). Except for the case $\omega=0$ and uniform backgrounds, this expansion does not lead to any secular term and can be used to compute the IV characteristic as shown in Refs. [12,13]. In the presence of noise, however, the secularity at $\omega=0$ implies a singularity in the low frequency part of the spectrum which could invalidate the calculation of the linewidth. To avoid this secularity we expand the solution of Eq. (4) as

$$
\Phi(x, t)=\Psi_{0}+\Psi_{\tilde{\chi}_{t}}(x, t)+\tilde{\psi}(x, t)+\tilde{\chi}(t),
$$

where $\Psi_{\tilde{\chi}_{t}}(x, t)$ is a quasideterministic modulation around $\Psi_{0}, \tilde{\chi}(t)$ is a stochastic homogeneous low frequency modulation of the phase, and $\tilde{\psi}(x, t) \ll 1$, the remaining part of the stochastic modulation. This splitting into slow and fast time components gives the extra variable $\tilde{\chi}(t)$, whose time evolution can be fixed to eliminate the secular term in the expansion. From a physical point of view it can be justified by assuming that the spectrum of the voltage consists mainly of two contributions, one at low frequency and the other around the Josephson frequency $\omega_{J}$ at which the system oscillates.

Substituting Eq. (7) into Eq. (4) and using the smallness of $\Psi_{\tilde{\chi}_{t}}(x, t)$ and $\tilde{\psi}(x, t)$ to linearize the resulting equation [note that no restriction is made on the magnitude of $\tilde{\chi}(t)$ ], we get

$$
\begin{aligned}
\eta-\left\{\alpha\left[\omega+\tilde{\chi}_{t}(t)\right]+\Psi_{\tilde{\chi}_{t}}(x, t) \cos \left[\Psi_{0}+\tilde{\chi}(t)\right]\right\}= & \left\{\left(-\partial_{x x}+\partial_{t t}+\alpha \partial_{t}\right) \Psi_{\tilde{\chi}_{t}}(x, t)+\sin \left[\Psi_{0}+\tilde{\chi}(t)\right]\right\} \\
& +\left[\left(-\partial_{x x}+\partial_{t t}+\alpha \partial_{t}\right) \tilde{\psi}(x, t)+\tilde{n}^{\prime}(x, t)+\tilde{n}_{H}(t)\right] \\
& +\tilde{n}_{L}(t)+\tilde{\psi}(x, t) \cos \left[\Psi_{0}+\tilde{\chi}(t)\right]+\tilde{\chi}_{t t}(t) .
\end{aligned}
$$

Here $\tilde{n}^{\prime}(x, t)$ represents the inhomogeneous part of the noise, which can be written as

$$
\tilde{n}^{\prime}(x, t)=\sum_{p=1}^{\infty} \tilde{n}_{p}(t) \cos \left(k_{p} x\right),
$$

with $k_{p}=\frac{\pi}{l} p,\left\langle\left\langle\tilde{n}_{p}(t)\right\rangle\right\rangle=0$, and

$$
\left\langle\left\langle\tilde{n}_{p}(t) \tilde{n}_{q}\left(t^{\prime}\right)\right\rangle\right\rangle=\left(2-\delta_{0, p}\right) \frac{16 \alpha k_{B} T}{E_{0} l} \delta_{p, q} \delta\left(t-t^{\prime}\right),
$$

while $\tilde{n}_{H}, \tilde{n}_{L}$, are, respectively, the high and the low frequency part of the homogeneous component of the noise $\tilde{n}_{0}(t)=\tilde{n}_{L}(t)+\tilde{n}_{H}(t)$. At zero temperature we have that all the tilde quantities vanish and $\Psi_{\tilde{\chi}_{t}}(x, t) \rightarrow \Psi(x, t)$, so that Eq. (8) reduces to

$$
\begin{aligned}
\eta-\alpha \omega+\Psi(x, t) \cos \left(\Psi_{0}\right)= & \left(-\partial_{x x}+\partial_{t t}+\alpha \partial_{t}\right) \Psi(x, t) \\
& +\sin \left(\Psi_{0}\right) .
\end{aligned}
$$

This is the same equation used in Refs. [12,13] to obtain the IV characteristic of a FFO in the absence of noise. We remark that one can solve Eq. (11) in two steps, i.e., by first computing the ac part [equating its right hand side (RHS) to zero], and then the dc part [equating its spacetime averaged left hand side (LHS) to zero]. The dc part gives just the IV curve. To generalize this to the case of nonzero noise, we note that the quasideterministic part of the phase can be obtained by equating the curly bracket in the RHS of Eq. (8) to zero and solving the corresponding 
equation for $\Psi_{\tilde{\chi}_{t}}(x, t)$. By inserting this solution into the space averaged LHS of Eq. (8), and equating it to zero, one gets

$$
\eta=\alpha\left(\omega+\tilde{\chi}_{t}\right)+\left\langle\Psi_{\tilde{\chi}_{t}}(x, t) \cos \left[\Psi_{0}+\tilde{\chi}(t)\right]\right\rangle,
$$

with \langle\rangle denoting the spatial average. In the following we assume that $\tilde{\chi}(t)$ is slow varying in comparison with the damping rate $\alpha$ of the high frequency oscillations (adiabatic approximation). In this case the frequency noise will follow the current noise and the IV curve can be obtained by averaging Eq. (12) in time. Since the quasideterministic part of the phase is not relevant for the calculation of the linewidth, we do not consider it further.

Once the quasideterministic terms in Eq. (8) are balanced, we can proceed with the balance of the low and high frequency stochastic components of the phase. To this end we remark that in Eq. (8) $\tilde{n}_{H}(t)$ acts as a noise source for the high frequency component $\tilde{\psi}(x, t)$, while $\tilde{n}_{L}(t)$ excites only the low frequency part, $\tilde{\chi}(t)$, of the phase. The nonhomogeneous high frequency part $\tilde{\psi}(x, t)$ can then be obtained by equating the square bracket in the RHS of Eq. (8) to zero, i.e.,

$$
\left(-\partial_{x x}+\partial_{t t}+\alpha \partial_{t}\right) \tilde{\psi}(x, t)+\tilde{n}^{\prime}(x, t)+\tilde{n}_{H}(t)=0 .
$$

By expanding $\tilde{\psi}(x, t)$ in Fourier modes $\tilde{\psi}(x, t)=$ $\sum_{p=0}^{\infty} \tilde{\psi}_{p}(t) \cos \left(k_{p} x\right)$, and using Eq. (9), we have that the spectrum of $\tilde{\psi}$ can be written as

$$
S_{\tilde{\psi}_{p}}= \begin{cases}\frac{S_{\tilde{n}_{H}}}{\omega^{2}\left(\omega^{2}+\alpha^{2}\right)} & \text { for } p=0 \\ \frac{S_{\tilde{n}_{p}}}{\left(\omega^{2}-k_{p}^{2}\right)^{2}+\alpha^{2} \omega^{2}} & \text { for } p=1,2, \ldots\end{cases}
$$

From Eq. (10) it follows that $S_{\tilde{n}_{p}}(\omega)=\left(2-\delta_{0, p}\right) S_{0}$, with $S_{0} \equiv 16 \alpha k_{B} T / E_{0} l$, so that no singularities appear in the spectrum of this part of the noise. On the other hand, from Eq. (14) we see that a singularity can arise at $\omega=0$ for $p=0$. The assumption made on the spectrum, however, allows the introduction of a limiting frequency $\omega_{0}$, which divides the spectrum in its high and low frequency components and which can be used to split the homogeneous part of the noise, for example, as

$$
\begin{aligned}
& S_{\tilde{n}_{H}}(\omega)=S_{0}\left(1-e^{-\frac{\omega^{2}}{\omega_{0}^{2}}}\right), \\
& S_{\tilde{n}_{L}}(\omega)=S_{0} e^{-\frac{\omega^{2}}{\omega_{0}^{2}}} .
\end{aligned}
$$

A condition for $\omega_{0}$ to hold is $\Delta \nu \ll \omega_{0} \ll \omega_{J}$. From Eq. (15) we have that at small frequencies $S_{\tilde{n}_{H}} \approx S_{0} \omega^{2} / \omega_{0}^{2}$ and, from Eq. (14), it follows that also the spectrum of $\tilde{\psi}_{0}$ has no singularity at zero frequency.

As the next step we balance the homogeneous low frequency components, i.e., all the terms not included in the curly and in the square brackets of Eq. (8). This leads, after averaging over space, to the following equation for the low frequency part:

$$
\begin{aligned}
& \eta-\alpha\left(\omega+\tilde{\chi}_{t}\right)-\left\langle\Psi_{\tilde{\chi}_{t}}(x, t) \cos \left[\Psi_{0}+\tilde{\chi}(t)\right]\right\rangle \\
& =\tilde{n}_{L}+\left\langle\tilde{\psi}(x, t) \cos \left[\Psi_{0}+\tilde{\chi}(t)\right]\right\rangle+\tilde{\chi}_{t t} .
\end{aligned}
$$

Because of the adiabatic approximation the last term in this equation can be neglected. Moreover, we can average Eq. (17) over fast time [16], thus leading to

$$
\eta-\eta\left(\omega+\tilde{\chi}_{t}\right)=\tilde{n}_{L}+\overline{\left\langle\tilde{\psi}(x, t) \cos \left[\Psi_{0}+\tilde{\chi}(t)\right]\right\rangle},
$$

where the overline denotes the fast time average and

$$
\eta\left(\omega+\tilde{\chi}_{t}\right) \equiv \alpha\left(\omega+\tilde{\chi}_{t}\right)+\overline{\left\langle\Psi_{\tilde{\chi}_{t}}(x, t) \cos \left[\Psi_{0}+\tilde{\chi}(t)\right]\right\rangle} .
$$

Note that the LHS of Eq. (18) can be rewritten as

$$
\eta-\eta\left(\omega+\tilde{\chi}_{t}(t)\right) \simeq-\left(\frac{\partial \omega}{\partial \eta}\right)^{-1} \tilde{\chi}_{t}(t),
$$

where $\partial \omega / \partial \eta \equiv r_{D}$ is the normalized dynamic resistance $\left(r_{D}=R_{D} / \alpha R\right)$. On the other hand, the RHS of Eq. (18) defines an effective noise

$$
\tilde{n}_{\text {eff }}=\tilde{n}_{L}+\overline{\left\langle\tilde{\psi}(x, t) \cos \left[\Psi_{0}+\tilde{\chi}(t)\right]\right\rangle}
$$

for the homogeneous low frequency part of the phase, so that Eq. (18) becomes

$$
\tilde{\chi}_{t}=-r_{D} \tilde{n}_{\mathrm{eff}} .
$$

From Eq. (3) we then see that the linewidth directly follows from the spectrum of $\tilde{n}_{\text {eff }}$ at zero frequency. Note that the second term in (21) represents a down-converted contribution originated from the noise around the Josephson frequency $\omega_{J}$. To compute this contribution, we use the fact that

$$
\cos \left[\Psi_{0}+\tilde{\chi}(t)\right]=\sum_{n=0}^{\infty}\left(2-\delta_{0, n}\right) \frac{\frac{\kappa l}{2}}{\frac{\kappa l+k_{n} l}{2}} \frac{\sin \frac{\kappa l-k_{n} l}{2}}{\frac{\kappa l-k_{n} l}{2}} \times \cos \left(k_{n} x\right) \cos \left[\omega_{J} t+\tilde{\chi}(t)+\frac{\kappa l-k_{n} l}{2}+\Phi_{0}\right] .
$$

Moreover, since the spectrum of $\tilde{\chi}_{t}$ is very narrow with respect to the characteristic size of the spectrum of $\tilde{\psi}$, we can neglect $\tilde{\chi}(t)$ in Eqs. (21) and (23) and compute the down-converted term as the mixing [17] of the stochastic signal $\tilde{\psi}$ with the deterministic one from Eq. (23). After some calculations, we get

$$
\begin{aligned}
S_{\tilde{n}_{\text {eff }}}(0) & =S_{\tilde{n}_{L}}(0)+\frac{1}{2} \sum_{n=0}^{\infty}\left[\frac{\frac{\kappa l}{2}}{\frac{\kappa l+k_{n} l}{2}} \frac{\sin \frac{\kappa l-k_{n} l}{2}}{\frac{\kappa l-k_{n} l}{2}}\right]^{2} S_{\tilde{\psi}_{n}}(\omega) \\
& =S_{0} \frac{\eta}{\alpha \omega},
\end{aligned}
$$

where, in the last step, we used the expression of the IV characteristic as derived in Refs. [12,13]. Since $\omega / \eta \equiv r_{S}$ 
is the normalized static resistance $\left(r_{S}=R_{S} / \alpha R\right)$, we have, from Eqs. (22) and (24), that

$$
S_{\tilde{\chi}_{t}}(0)=S_{0} \frac{r_{D}^{2}}{\alpha r_{S}} .
$$

From this expression and from Eq. (3) it follows, after restoring physical dimensions [18], that the linewidth of a FFO is given by Eq. (1) with the prefactor $\rho=1$.

From the above derivation it is clear that the factor $1 / R_{S}$ in Eq. (1) is due to the down-converted term in the effective noise, and it represents the contribution of the FFO dynamics to the linewidth expression (if only $\tilde{n}_{L}$ would be present in $\tilde{n}_{\text {eff }}$, one would get instead of $1 / R_{S}$, the reciprocal normal resistance characterizing just the Ohmic part of the IV characteristic).

Before closing this paper we discuss the assumptions made and the range of validity of the result. Our approach is based on the ansatz solution in Eq. (7) and on the adiabatic approximation. Moreover, we assumed a Lorentzian limit for the validity of Eq. (3). A main assumption, however, was made on the form of the spectrum for which we considered only two main contributions, one around the Josephson frequency, related to the flux-flow dynamics, and the other at very low frequencies, related to the fluctuations of the homogeneous background rotations. From a physical point of view, this is quite reasonable since one expects that contributions to the spectrum coming from Fiske steps would influence the linewidth only through second order effects, i.e., down-conversions from $\omega_{J}$ to Fiske frequencies which are then further down-converted to zero. Since Fiske steps have a width $\approx \alpha$ and are spaced in frequency by multiples of $\pi / l$, to avoid overlappings one must require

$$
\Delta \nu \ll \omega_{0}<\alpha \ll \frac{\pi}{l} \ll \omega_{J}
$$

Although these conditions appear restrictive, they are satisfied by most Josephson devices.

We finally remark that in comparison with the experimental results of Ref. [5] there is a factor $\approx 8$ missing in our formula. This discrepancy could be due to the appearance of noise-induced parametric excitations (not included in our analysis), leading to an increased effective temperature in the system. This possibility could be experimentally checked by investigating the linewidth dependence on the temperature (the experiments reported in Refs. [5] were done at fixed temperatures). Another excess of noise source in the system could be the presence of deterministic chaos, a phenomenon which was indeed observed in the numerical simulations of a FFO in the absence of noise [19].
In conclusion, we have shown that in spite of the different internal dynamical states characterizing different types of Josephson oscillators, under certain conditions, a universal formula for the linewidth of Josephson oscillators emerges.

We acknowledge discussions with V.P. Koshelets, J. Mygind, and A. L. Pankratov.

*Permanent address: Dipartimento di Fisica "E.R. Caianiello," and Istituto Nazionale di Fisica della Materia (INFM), Universita' di Salerno, I-84100 Salerno, Italy. Email address: salerno@sa.infn.it

[1] T. Nagatsuma, K. Enpuku, F. Irie, and K. Yoshida, J. Appl. Phys. 54, 3302 (1983); 56, 3284 (1984).

[2] A. V. Ustinov, J. Mygind, and V. A. Oboznov, J. Appl. Phys. 72, 1203 (1992).

[3] A. V. Ustinov, J. Mygind, N.F. Pedersen, and V. A. Oboznov, Phys. Rev. B 46, 578 (1992).

[4] V.P. Koshelets, A. V. Shchukin, S. V. Shitov, and L. V. Filippenko, IEEE Trans. Appl. Supercond. 3, 2524 (1993).

[5] J. Mygind, V. P. Koshelets, A. V. Shchukin, S. V. Shitov, and I. L. Lapitskaya, IEEE Trans. Appl. Supercond. 5, 2951 (1995); V. P. Koshelets, A. V. Shchukin, I. L. Lapytskaya, and J. Mygind, Phys. Rev. B 51, 6536 (1995).

[6] K. K. Likharev, Dynamics of Josephson Junction and Circuits (Gordon and Breach, New York, 1986), and references therein.

[7] A. J. Dahm, A. Denenstein, D. N. Langenberg, W. H. Parker, D. Rogovin, and D. J. Scalapino, Phys. Rev. Lett. 21, 1629 (1968).

[8] M. J. Stephen, Phys. Rev. Lett. 22, 1416 (1969).

[9] E. Joergensen, V. P. Koshelets, R. Monaco, J. Mygind, M. R. Samuelsen, and M. Salerno, Phys. Rev. Lett. 49, 1093 (1982).

[10] A. A. Golubov, B. A. Malomed, and A. V. Ustinov, Phys. Rev. B 54, 3047 (1996).

[11] A. P. Betenev and V. V. Kurin, Phys. Rev. B 56, 7855 (1997).

[12] M. Cirillo, N. Gronbech-Jensen, M. R. Samuelsen, M. Salerno, and G. Verona Rinati, Phys. Rev. B 58, 12377 (1998).

[13] M. Salerno and M. R. Samuelsen, Phys. Rev. B 59, 14653 (1999).

[14] M. Salerno and M. R. Samuelsen, Phys. Rev. B 61, 99 (2000).

[15] J. L. Stewart, Proc. IRE 42, 1539 (1954).

[16] This average eliminates the fast oscillations but keeps the low frequency ones and is defined as $\bar{f}=\omega_{0} \int_{-\infty}^{t} f\left(t^{\prime}\right) \exp \left[\omega_{0}\left(t^{\prime}-t\right)\right] d t$.

[17] Use the fact that for a signal of type $\tilde{X}(t)=\tilde{x}(t) \cos (\Omega t)$ the power spectrum is $S_{\tilde{X}}(\omega)=\frac{1}{2}\left[S_{\tilde{x}}(\omega+\Omega)+S_{\tilde{x}}(\omega-\right.$ $\Omega)]$.

[18] Note that Eq. (3) is in normalized units and that $S_{0}$ in physical units is $4 \pi k_{B} T \frac{2 \pi}{\omega_{J}} \frac{\alpha^{2} R}{\phi_{0}^{2}}$.

[19] M. Salerno and M. R. Samuelsen (unpublished). 